\title{
Virtual Visits and the Future of No-Shows
}

\author{
Nathaniel P. Morris, MD \\ Department of Psychiatry and Behavioral Sciences, Stanford University School of Medicine 401 Quarry Road, Stanford, CA, USA
}

J Gen Intern Med 35(8):2449-50

DOI: $10.1007 / \mathrm{s} 11606-020-05948-2$

(c) Society of General Internal Medicine 2020

${ }^{66} \mathrm{t}$ was great to see you, and keep washing those hands."

"You too, Doc!"

The patient's finger approaches the screen. The screen goes black as his face and the interior of his car disappears. I had never completed a clinic appointment with a patient in a parked car until a few weeks ago. Now, as our clinic shifts to virtual visits amid the coronavirus disease 2019 (COVID19) pandemic, I find myself routinely talking with patients in their cars, their closets, and their backyards. At first, these virtual visits seemed awkward and clunky. Patients shouted into their smartphones, and I shouted at my computer as we tried to hear one another. I couldn't help but notice patients' belongings strewn in their backseats, the coats hanging over them, and the neighborhoods around them.

Then I realized something startling. Despite these logistical issues, I couldn't recall the last time I had a no-show.

No-shows, or when patients miss healthcare appointments, are the underbelly of medicine. Estimates of outpatient noshow rates vary widely, with healthcare institutions reporting rates between 9 and $76 \%$ in recent years. ${ }^{1-4}$ In 2008 , the Veterans Health Administration reported approximately 3.1 million (12\%) no-shows out of 26.5 million scheduled outpatient appointments and an estimated annual cost of 564 million from the total number of unused appointments. ${ }^{1}$ No-shows can lead to disruptions in patient-clinician relationships, interruptions in pharmacotherapy, lack of testing follow-up, greater utilization of higher levels of care, psychological stress, and other adverse outcomes for patients.

Clinics can introduce measures, such as pre-appointment phone or text reminders, that may decrease no-show rates. However, in-person clinic visits still pose obstacles that can be challenging, if not insurmountable, for patients. In addition to keeping track of appointment locations and timing, patients and families may need to travel lengthy distances to clinics, which may entail taking public transportation, finding a ride,

Received May 12, 2020

Accepted May 28, 2020

Published online June 8, 2020 driving, walking, or a combination of modes of travel. Upon arrival, patients may need to find and pay for parking, find the right clinic, find the right waiting room, and check in at the front desk. Then, patients may sit and wait, often with prolonged delays, until someone brings them to a patient room. Support staff might ask basic questions or take vitals, but then patients may wait for longer periods until their clinicians arrive at the appointments. After completing their visits, which may last just minutes, patients then return to the waiting room, check out, and complete the whole process in reverse. Other factors, including disabilities and language barriers, may make it harder for patients to attend in-person visits. A study from Ireland of over 200 patients at general surgery clinics in 2018 estimated that one-way travel from home to clinic and waiting for a clinician took $1 \mathrm{~h} 44$ min on average. ${ }^{2}$

By allowing patients to remotely attend appointments through audiovisual applications, virtual visits bring promise for decreasing outpatient no-shows. Patients can attend virtual visits from anywhere, including their homes, their workplaces, or even their parked cars, decreasing the burdens of making it to in-person appointments. In a 2016 survey of 218 patients who missed primary care appointments, $15(7 \%)$ reported problems with transportation, and $82(38 \%)$ reported either forgetting or not knowing about the appointment. ${ }^{5}$ Since virtual visits already involve electronic applications and messaging for setup, these systems can integrate automated reminders, such as email or mobile notifications, to enhance appointment attendance. Patients can $\log$ in at the start times of their visits or when their clinicians are ready, which may reduce unnecessary waiting. A 2017 randomized trial of 195 patients with Parkinson's disease found home virtual visits saved patients a median of $88 \mathrm{~min}$ and 38 miles compared with usual care. ${ }^{6}$ In addition to convenience, patients may prefer virtual visits for other reasons, such as increased access to virtual interpreters or privacy around potentially sensitive topics. For instance, some patients might hesitate to walk into clinics with signs for psychiatry or addiction treatment, but they might be willing to virtually meet with a clinician to discuss these health concerns.

Virtual visits are not be a universal solution for no-shows. Patients with limited socioeconomic resources, limited Internet access, ognitive impairment, or other physical disabilities might not be able to use digital technologies required for virtual visits. Some patients might have difficulty accessing separate spaces for speaking privately with clinicians during virtual visits. In-person appointments will be necessary for 
some patients with medical needs that virtual visits cannot adequately address and, in addition, patients might opt not to participate in virtual visits, for example, due to preferences for in-person appointments or concerns about digital privacy. In the study of general surgery outpatients from 2018, $123(55 \%)$ of 223 patients reported they would prefer in-person appointments over virtual ones, even when accounting for the time and cost of coming to in-person appointments. ${ }^{2}$ Patients and clinicians might struggle with setting up the technology needed for virtual visits, which might waste time and resources. If virtual visits require less investment for patients to attend, or if patients believe that virtual no-shows are less disruptive to health systems, patients may be more likely to miss these appointments. Finally, virtual visits might not change attendance if patients would not come anyways due to poor relationships with clinicians or mistrust of healthcare. In the 2016 survey of patients who missed primary care appointments, 9 (4\%) of 218 reported not coming due to negative experiences with their physicians or the practice. ${ }^{5}$

More research is needed to clarify how virtual visits influence no-show rates. For example, a 2019 study from Canada of home virtual visits for 75 patients at a stroke prevention clinic reported a no-show rate of $2(3 \%)$ out of 81 appointments, but the study did not include comparisons of no-show rates for in-person clinic visits. ${ }^{7}$ At a 2019 conference, researchers reported preliminary findings from a randomized trial of 108 patients receiving follow-up after hospitalization for heart failure; virtual visits were associated with a greater than $30 \%$ reduction in no-show rates compared with in-person visits, although this association did not reach statistical significance. $^{3}$

The COVID-19 pandemic may prompt a shift in outpatient care toward virtual visits for the foreseeable future, and noshow rates are far from the only outcomes that need further study during this transition. Health professionals and patients are facing uncertainties about their comfort with virtual visits, best practices for using these technologies, when in-person visits may be necessary, and the effectiveness of virtual visits compared with usual care. Still, as social distancing efforts seek to keep people apart, perhaps this pandemic might help health professionals better recognize the everyday obstacles, from transportation burdens to wait times, that prevent patients from making it to appointments. Whether during a pandemic or not, simply connecting with our patients, and maintaining our relationships with them, may be what matters most.

Seeing patients in their parked cars may not be a perfect way of providing care. But isn't it better than patients not coming at all?

Corresponding Author: Nathaniel P. Morris, MD; Department of Psychiatry and Behavioral Sciences, Stanford University School of Medicine 401 Quarry Road, Stanford, CA 94305, USA (e-mail:npm@stanford.edu).

\section{Compliance with Ethical Standards:}

Conflict of Interest: The author declares that he does not have a conflict of interest.

\section{REFERENCES}

1. Department of Veterans Affairs Office of Inspector General. Audit of Veterans Health Administration's Efforts to Reduce Unused Outpatient Appointments. December 4, 2008. Available at: https://www.va.gov/oig/ 52/reports/2009/VAOIG-08-00879-36.pdf. Accessed April 3, 2020.

2. Rutherford E, Noray $\mathbf{R}$, Ó HEarráin $\mathbf{C}$, et al. Potential benefits and drawbacks of virtual clinics in general surgery: pilot cross-sectional questionnaire study. JMIR Perioper Med. 2020;3:e12491.

3. Gorodeski EZ, Moennich LA, Riaz H, Tang WHW. Virtual visits versus inperson visits and appointment no-show rates. J Card Fail. 2019;25:939.

4. Kheirkhah P, Feng $\mathbf{Q}$. Travis LM, Tavakoli-Tabasi S, Sharafkhaneh $\mathbf{A}$ Prevalence, predictors and economic consequences of no-shows. BMC Health Serv Res. 2016;16:13.

5. Saif U, Sangeetha R, Todd L, et al. Why do patients miss their appointments at primary care clinics? J Fam Med Dis Prev. 2018;4:090.

6. Beck CA, Beran DB, Biglan KM, et al. National randomized controlled trial of virtual house calls for Parkinson disease. Neurology. 2017;89:11521161.

7. Appireddy R, Khan S, Leaver C, et al. Home virtual visits for outpatient followup stroke care: cross-sectional study. J Med Internet Res. 2019;21:e13734.

Publisher's Note: Springer Nature remains neutral with regard to jurisdictional claims in published maps and institutional affiliations. 